\title{
AMENDMENTS
}

\section{Author Correction: Robust SARS-CoV-2-specific T cell immunity is maintained at 6 months following primary infection}

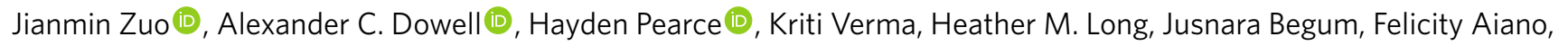
Zahin Amin-Chowdhury, Katja Hoschler, Tim Brooks, Stephen Taylor, Jacqueline Hewson, Bassam Hallis, Lorrain Stapley,

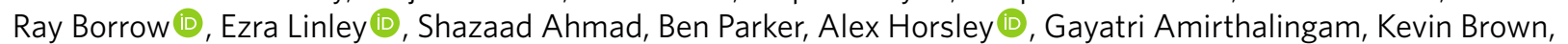
Mary E. Ramsay, Shamez Ladhani and Paul Moss $\mathbb{B}$

Correction to: Nature Immunology https://doi.org/10.1038/s41590-021-00902-8, published online 5 March 2021.

In the version of this article initially published, authors Katja Hoschler, Tim Brooks, Stephen Taylor and Jacqueline Hewson were omitted from the author list, and their contributions were omitted from the Author Contribution statement. Their contributions (initials in bold) are as follows: "F.A., Z.A.-C., K.H., L.S., R.B., E.L., S.A., B.P., A.H., G.A., K.B., M.R. and S.L. devised, set up and recruited the ESCAPE cohort. T.B., S.T., J.H. and B.H. performed humoral analyses." Also, the following sentence was omitted from the beginning of the Acknowledgements: "The authors would like to thank the staff in the Virus Reference Division at PHE Colindale, who provided testing and contributed to antibody assay development." The errors have been corrected in the HTML and PDF versions of the article.

Published online: 20 May 2021

https://doi.org/10.1038/s41590-021-00957-7

( ) The Author(s), under exclusive licence to Springer Nature America, Inc. 2021

\section{Author Correction: Trained immunity, tolerance, priming and differentiation: distinct immunological processes}

Maziar Divangahi@i], Peter Aaby, Shabaana Abdul Khader (1D, Luis B. Barreiro, Siroon Bekkering (D), Triantafyllos Chavakis (D), Reinout van Crevel, Nigel Curtis, Andrew R. DiNardo, Jorge Dominguez-Andres (1), Raphael Duivenvoorden, Stephanie Fanucchi, Zahi Fayad (1D, Elaine Fuchs, Melanie Hamon (D), Kate L. Jeffrey (D), Nargis Khan, Leo A. B. Joosten (D), Eva Kaufmann, Eicke Latz (D), Giuseppe Matarese (1), Jos W. M. van der Meer, Musa Mhlanga (D), Simone J. C. F. M. Moorlag, Willem J. M. Mulder (1), Shruti Naik, Boris Novakovic, Luke O'Neill (D, Jordi Ochando (1), Keiko Ozato, Niels P. Riksen (D), Robert Sauerwein, Edward R. Sherwood, Andreas Schlitzer, Joachim L. Schultze, Michael H. Sieweke, Christine Stabell Benn, Henk Stunnenberg, Joseph Sun (1), Frank L. van de Veerdonk (1D, Sebastian Weis (1D), David L. Williams, Ramnik Xavier (1) and Mihai G. Netea (1)

Correction to: Nature Immunology https://doi.org/10.1038/s41590-020-00845-6, published online 8 December 2020.

In the version of this article initially published, author Raphael Duivenvoorden's last name was spelt incorrectly as Duivenwoorden. The error has been corrected in the HTML and PDF versions of the article.

Published online: 20 May 2021

https://doi.org/10.1038/s41590-021-00960-y

๑) Springer Nature America, Inc. 2021 